\title{
Protocol
}

\section{An Assay for Visual Learning in Individual Drosophila Larvae}

\author{
Bruno van Swinderen
}

Vision is a major sensory modality in Drosophila behavior, with more than one-half of the Drosophila brain devoted to visual processing. The mechanisms of vision in Drosophila can now be studied in individuals and in populations of flies by using various paradigms. Simple strategies for conducting visual perception and learning studies consist of individual studies performed on single flies on solid supports (larvae on agar or adults in a T-maze) using a light/dark association paradigm. These approaches are quite easy to implement but are fairly limited in their ability to address questions of visual perception. Nevertheless, the simpler approaches treating vision in one dimension (light, dark) do provide effective paradigms for genetic analysis. This article describes a protocol for larval visual learning. Larvae are transferred back and forth between well-lit or dark agarose plates that either do or do not contain fructose (which is appetitive) as the unconditioned stimulus (US). Such appetitive larval visual memory is tested by tallying the time spent in light or dark quadrants of a plate (without a US).

Protocols for larval visual learning can be found in Gerber et al. (2004) and Kaun et al. (2007). For a simple assay for adult visual learning, see The Aversive Phototaxic Suppression Assay for Individual Adult Drosophila (van Swinderen 2011).

It is essential that you consult the appropriate Material Safety Data Sheets and your institution's Environmental Health and Safety Office for proper handling of equipment and hazardous materials used in this protocol.

Reagents

Agarose (electrophoresis grade)

Drosophila larvae, aged $90-115 \mathrm{~h}$ since a 24 -h batch of egg-laying

Fructose to be used as the US

Equipment

Aluminum foil (for use as a shielding device; see Step 2)

Brush, fine

Cardboard (for use as a shielding device; see Step 2)

Adapted from Drosophila Neurobiology (ed. Zhang et al.). CSHL Press, Cold Spring Harbor, NY, USA, 2010.

(c) 2011 Cold Spring Harbor Laboratory Press

Cite this article as Cold Spring Harbor Protoc; 2011; doi:10.1101/pdb.prot065888 
Cold-light boxes such as those used for visualizing radiographs (two)

Petri dishes, plastic, 9-cm diameter

1. Prepare plates:

i. Prepare a $1 \%$ agarose solution in water. Boil.

ii. Add fructose (1 M final) $10 \mathrm{~min}$ after boiling.

iii. Pour a thin layer into the Petri dishes, and let cool.

iv. Also prepare plates with agarose only.

For a sample size of one, prepare 20 fructose-containing plates ( 10 for each of the reciprocally trained larvae; see below) and 22 agarose-only plates (10 each for training, 1 each for testing), as fresh plates must be used for every trial.

2. Prepare shielding devices:

i. For training: Prepare light box shields for light and heat by covering one of the light boxes with black cardboard shielded from below by aluminum foil to prevent heating up of the cardboard.

ii. For testing: Prepare an X-mask made of foil/cardboard so as to shield two opposing quadrants of the Petri dish, allowing light through the other two quadrants (see Fig. 1).

3. Using a fine brush, collect 90-115-h larvae from a food bottle, and gently wash them with tap water.

4. For training, ensure that the room is dark, with the only light coming from the noncovered light box. Below, a sample training regimen is detailed in which larvae associate light with reward and are placed in the dark without reward (light+/dark):

i. Place a larva on a fructose plate.

ii. Set the plate on the noncovered light box for $1 \mathrm{~min}$.

iii. Transfer the larva from the fructose plate onto a fresh plate with agarose only, and place it on the covered light box.

iv. Repeat Steps 4.i-iii 10 times.

5. For testing:

i. Put the X-mask onto the noncovered light box.

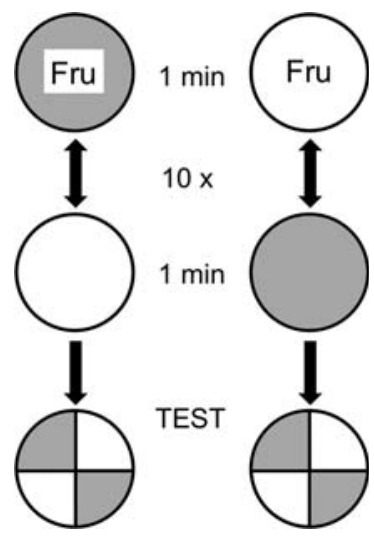

FIGURE 1. Larvae are transferred from darkened plates (gray) with fructose to lit plates (white) without fructose, or the converse. An average of both experiments is performed to yield a learning score. 
B. van Swinderen

ii. Transfer the trained larva to test plates that contain agarose only, and place them onto the $\mathrm{X}$-mask of the light box.

iii. Score the position of the larva every $10 \mathrm{sec}$ for $5 \mathrm{~min}$, as in light or dark.

6. Calculate a first preference value as PREF1 $=[$ (counts in light $)-($ counts in dark $)] /($ total counts).

7. Perform the reciprocal experiment (light/dark+) following Steps 1-5 and using another larva and fresh plates. Calculate a second preference value as PREF2 $=[$ (counts in light $)-($ counts in dark)]/(total counts).

8. Calculate a learning index $(\mathrm{LI})$ as $\mathrm{LI}=(\mathrm{PREF} 1-\mathrm{PREF} 2) / 2$, and analyze the data with nonparametric statistics.

9. Perform the experiment for both reciprocal regimens following Steps 1-6 and using the respective other sequence of trials (i.e., dark/light+ and dark+/light, respectively).

The duration of the training trials may also be 3 min (Kaun et al. 2007). The experiment may be multiplexed by training a group of larvae, but testing them individually, if multiple light boxes are available for testing, or by performing the tests in smaller diameter Petri dishes using $0.5 \times 0.5-\mathrm{cm}$ checkerboard masks. Whether a mass version of the assay is feasible and whether aversive learning may be uncovered by performing the test in the presence of an aversive reinforcer (as has been shown for olfactory learning [Gerber and Hendel 2006]) remain to be tested.

\section{REFERENCES}

Gerber B, Hendel T. 2006. Outcome expectations drive learned behaviour in larval Drosophila. Proc Biol Sci 273: 2965-2968.

Gerber B, Scherer S, Neuser K, Michels B, Hendel T, Stocker RF, Heisenberg M. 2004. Visual learning in individually assayed Drosophila larvae. J Exp Biol 207: 179-188.
Kaun KR, Hendel T, Gerber B, Sokolowski MB. 2007. Natural variation in Drosophila larval reward learning and memory due to a cGMPdependent protein kinase. Learn Mem 14: 342-349.

van Swinderen B. 2011. The aversive phototaxic suppression assay for individual adult Drosophila. Cold Spring Harb Protoc doi: 10.1101/ pdb.prot065896. 


\section{An Assay for Visual Learning in Individual Drosophila Larvae}

Bruno van Swinderen

Cold Spring Harb Protoc; doi: 10.1101/pdb.prot065888

\begin{tabular}{cc}
$\begin{array}{c}\text { Email Alerting } \\
\text { Service }\end{array}$ & Receive free email alerts when new articles cite this article - click here. \\
\hline $\begin{array}{c}\text { Subject } \\
\text { Categories }\end{array}$ & $\begin{array}{c}\text { Browse articles on similar topics from Cold Spring Harbor Protocols. } \\
\text { Behavioral Assays (99 articles) } \\
\text { Drosophila (272 articles) } \\
\text { Neuroscience, general (357 articles) }\end{array}$ \\
\hline
\end{tabular}

